\title{
Angular distribution of prompt neutrinos in Extensive Air Showers
}

\section{A. Müller*}

Instituto de Física e Matemática, Universidade Federal de Pelotas/UFPEL, Pelotas, RS, Brazil and Instituto de Física, Universidade Federal do Rio de Janeiro/UFRJ, Rio de Janeiro, Brazil

E-mail: mamullereif.ufrj.br

\section{Victor P. Gonçalves}

Instituto de Física e Matemática, Universidade Federal de Pelotas/UFPEL, Pelotas, RS, Brazil

E-mail: barros@ufpel.edu.br

\begin{abstract}
Atmospheric neutrinos are produced in interactions of Cosmic Rays with the Earth's atmosphere and are an important background to other sources of neutrinos, such as the cosmogenic neutrinos. The flux of very high energy leptons is directly associated to the heavy quark (charm and bottom) production in the Extensive Air Showers (EASs), with the prompt decay of charmed hadrons being expected to be the dominant source of leptons at $\mathrm{PeV}$ energies. In this contribution we estimate the prompt neutrino flux considering a modified version of the CORSIKA Monte Carlo, which includes the production of charmed particles in the first interaction of the primary cosmic ray. The heavy quark production is modelled considering two different models: the Color Glass Condensate (CGC) formalism and the Intrinsic Quark Model (IQM). The modified version of the code allows us to analyze the impact of the production and propagation of heavy hadrons in the EAS development. In particular, we will present our results for the angular and energy distributions of the prompt neutrinos in the EASs. We will present the CGC and IQM predictions considering different values for the zenithal angle of the primary particle. Energy cuts are applied $(1 \mathrm{PeV})$ in order to analyze the most energetic neutrinos. We demonstrate that such neutrinos follow the direction of the primary particle. Such result is useful to estimate the number of high energy leptons that reach the ground.
\end{abstract}

36th International Cosmic Ray Conference -ICRC2019-

July 24th - August 1st, 2019

Madison, WI, U.S.A.

* Speaker. 


\section{Introduction}

A precise description of Extensive Air Showers (EASs) is fundamental for the Cosmic Ray Physics. Primary particles reach the Earth with energies up to $10^{20} \mathrm{eV}$. For such energies, very energetic heavy hadrons, composed by charm and bottom quarks, can be produced in the upper atmosphere when a primary cosmic ray or leading hadron in an EASs collides with the air. The decay of these hadrons can generated an additional contribution for the atmospheric neutrino flux, denoted prompt neutrino flux. In this contribution we will study the production and decay of charmed particles in the EAS development.

The atmospheric flux of neutrinos at low energies, denoted conventional neutrino flux, has been extensively studied in literature (See, e.g. Refs. [1, 2]). It arise mainly from the decay of charged pions and kaons. The increasing of the energy of the primary cosmic ray implies that the decay lenght of the mesons become larger than their mean path lenghts, suppressing the production of muons and neutrinos. For energies above $100 \mathrm{GeV}$, pions and kaons become less effective in the production of atmospheric muons and neutrinos, since they tend to collide with air nuclei instead of decaying [3]. At large energies, the charmed meson production becomes non- negligible and the decay of these particles starts to contribute for the muon and neutrino fluxes, especially from the "prompt" decay of charmed mesons at PeV energies. The energy dependence of these prompt neutrinos is less steep than the conventional neutrino flux from the pion and kaon decays. Therefore, the flux of high energy leptons in EASs is directly associated to the charm production. In fact, the prompt decay of charmed hadrons has been extensively studied [4, 5], being predicted to be the dominant source of leptons at PeV energies.

In this contribution we will estimate the prompt muon and neutrino fluxes considering a modified version of the CORSIKA (Cosmic Ray Simulations for Kaskade) Monte Carlo [6], hereafter denoted HQ CORSIKA, which includes the charm and bottom production at the cosmic ray first interaction (For more details see Refs. [7, 8]. In order to estimate the impact of the heavy particles in the EAS development, we will compare our predictions with those derived using the standard version of the CORSIKA (STD CORSIKA). In both cases, the high energy hadronic interactions will be described by the QGSJET01 model. We will focus on the analysis of the zenithal distribution of the prompt neutrinos generated in the EASs. We will present results considering that the heavy hadron production is described by the Color Glass Condensate (CGC) formalism or by the Intrinsic Quark Model (IQM). Our goal is to estimate the amount of the high energy neutrinos, with energy above $1 \mathrm{PeV}$, and the zenithal angle that such particles hit the ground. Considering distinct values for the zenithal angle of the incident primary particle $\left(0^{\circ}, 15^{\circ}, 30^{\circ}, 45^{\circ}\right.$ and $\left.60^{\circ}\right)$ we will estimate the flux of prompt leptons and the $\mu, v_{\mu}$ and $v_{e}$ energy distributions.

\section{Prompt lepton flux}

The recent detection of ultra-high energy neutrino events with deposited energies up to a few $\mathrm{PeV}$ by the IceCube Observatory sets the begining of neutrino astronomy $[9,10,11]$. In order to advance in this new area, it is mandatory to know the flux of atmospheric neutrino produced in cosmic-ray interactions with nuclei in Earth's atmosphere at different energies with high precision, which is an unavoidable background for cosmic neutrino studies. 
The available data indicate that the neutrino flux observed in the experiment is dominated at low energies $\left(E_{v} \lesssim 10^{5} \mathrm{GeV}\right)$ by atmospheric neutrinos that arise from the decay of light mesons (pions and kaons), denoted as the conventional atmospheric neutrino flux while the data for the higher energies $\left(E_{v} \gtrsim 10^{7} \mathrm{GeV}\right)$ are most probably associated with cosmic neutrinos. In the intermediate energy range $\left(10^{5} \mathrm{GeV}<E_{V}<10^{7} \mathrm{GeV}\right)$, it is expected that the prompt atmospheric neutrino flux associated with the decay of heavy flavoured hadrons, composed of heavy quarks, become important. In particular, it is typically considered that this contribution dominates the atmospheric neutrino flux for large neutrino energies $\left(E_{v}>10^{6} \mathrm{GeV}\right)$. Associated to this prompt neutrino flux, we also expect the presence of a prompt muon flux.

At very high energies, the atmospheric lepton flux is expected to arise from semi-leptonic decays of heavy, in particular charmed hadrons. Thus, the precise knowledge of the prompt lepton flux is crucial for the determination of the cosmic neutrino flux. This subject has been a theme of intense debate in the literature, mainly due to the fact that the calculation requires good knowledge of the heavy quark production cross section at high energies. In principle, for high energies, the charm cross section is sensitive to the presence of non - linear effects in the QCD dynamics [12, 5], as described by the Color Glass Condensate formalism. Moreover, it can also be modified by an intrinsic heavy quark component in the hadron wave function $[13,14]$. Such alternatives have been implemented in a modified version of the CORSIKA Monte Carlo, denoted HQ CORSIKA, which will be used to estimate the prompt lepton flux and the zenithal angle distributions.

\section{Energy and angular distributions of the prompt leptons}

In this section we present our results for the energy distribution of the prompt leptons considering EASs simulated assuming a primary proton with energy of $3 \times 10^{19} \mathrm{eV}$ and several zenithal angles $\left(0^{\circ}, 15^{\circ}, 30^{\circ}, 45^{\circ}\right.$ and $\left.60^{\circ}\right)$. The events are simulated using the HQ CORSIKA, assuming that the heavy quark production is described by CGC formalism or the Intrinsic Quark Model. Moreover, we will assume that The level of observation of the particles is positioned at the sea level. For completeness we also will present the standard CORSIKA predictions, which disregard the presence of heavy particles in the EAS development.

In the Figs. 1, 2 and 3 we present our results for the prompt $v_{e}, v_{\mu}$ and $\mu$ distributions, respectively, considering some values of the zenithal angle of the primary particle $\left(0^{\circ}, 15^{\circ}, 30^{\circ}\right.$, $45^{\circ}$ and $60^{\circ}$ ). Our results indicate that the presence of the heavy particles in the EASs development implies an enhancement of the prompt lepton fluxes at PeV energy. Such results occurs for both models of heavy quark production (CGC and IQM). Regarding prompt muon flux, presented in Fig. 3, the excess starts at $\approx 10^{5} \mathrm{GeV}$ and reaches a maximum for $E \approx 10^{8} \mathrm{GeV}$. On the other hand, in the case of the prompt neutrino flux, we have an excess starting for $E \approx 10^{5} \mathrm{GeV}$ to $v_{\mu}$ and $\approx 10^{4} \mathrm{GeV}$ to $v_{e}$ and being maximum for $E \approx 10^{8} \mathrm{GeV}$. Therefore, the presence of heavy particles implies that the prompt lepton fluxes are amplifies for $\mathrm{PeV}$ energies in cosmic ray interactions in the atmosphere. Comparing now the flux generated by different production models, we have that the IQM model implies a a less steep curve. In general, our predictions for the prompt leptons are less steep than the conventional lepton flux. Therefore, the prompt lepton flux is dominant in comparison to the conventional one for $\mathrm{PeV}$ energies. In addition, we have that this enhancement at large energies is present for all zenithal angles, being larger for more inclined EASs $\left(60^{\circ}\right)$. 

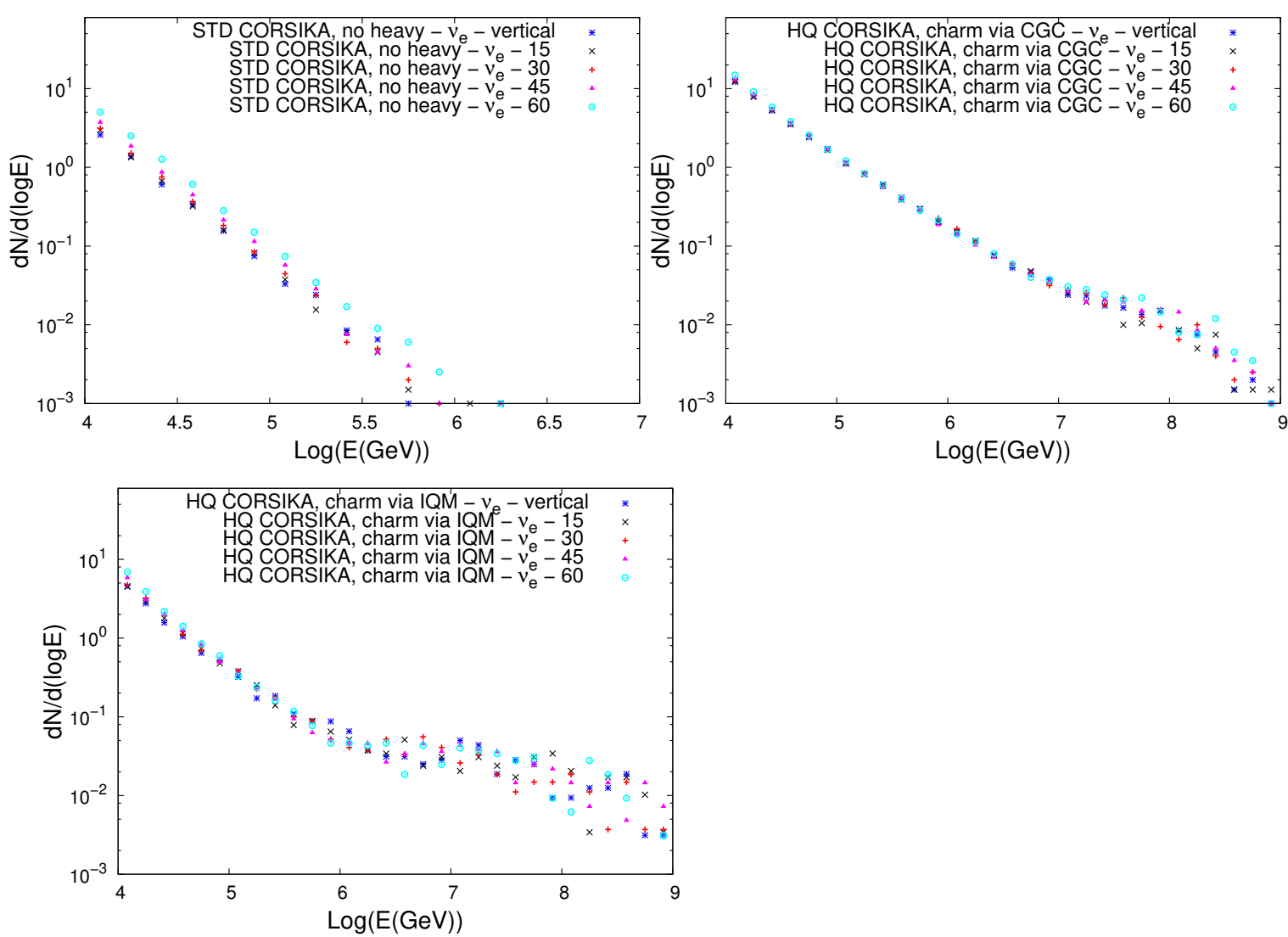

Figure 1: Energy distribution for the prompt $v_{e}$ flux disregarding the heavy particles in the EASs development (top left panel) and including these particles considering the CGC formalism (top right panel) and Intrinsic Quark Model (lower panel) to describe the heavy quark production. Results for 1000 EASs.

Let's now present our results for the zenithal angular distributions of the electron and muon neutrinos that reach the ground with energy larger than $1 \mathrm{PeV}$, considering the observation at the sea level. We consider that the primary proton has a energy of $3 \times 10^{19} \mathrm{eV}$. The analysis will be performed considering different values of the zenithal angle of the primary particle and we will compare the HQ and STD CORSIKA predictions. For the heavy quark production we will assume the CGC formalism, but similar results are obtained for the IQM one. In the Table 1 we present our predictions for the average number of electron and muon neutrinos derived considering different values of the zenithal angle of the primary proton. If the heavy particles are not included in the EAS development, the STD CORSIKA predicts a small number of neutrinos with energy larger than 1 $\mathrm{PeV}$. On the other hand, including the heavy quark contribution, the average number is increased by a factor larger than 15. Moreover, we have verified that the prompt contribution is larger for more inclined showers and that the main contribution comes from the neutrinos that are in the direction of the primary particle. Thus we can say that prompt neutrinos have the same zenithal angle that the source of the EAS. 

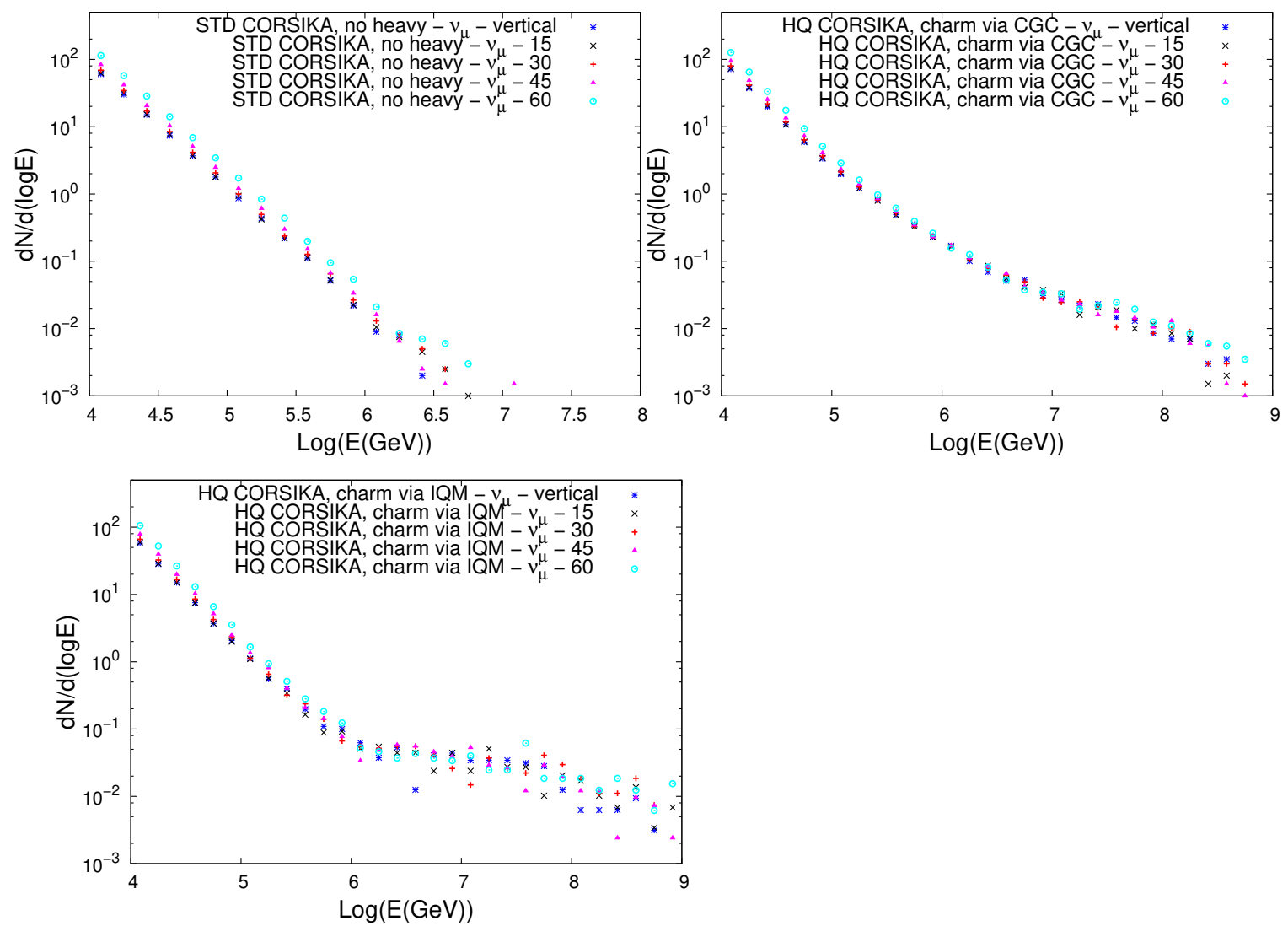

Figure 2: Energy distribution for the prompt $v_{\mu}$ flux disregarding the heavy particles in the EASs development (top left panel) and including these particles considering the CGC formalism (top right panel) and Intrinsic Quark Model (lower panel) to describe the heavy quark production. Results for 1000 EASs.

\begin{tabular}{|c|c|c|c|c|c|}
\hline & $\theta=0^{\circ}$ & $\theta=15^{\circ}$ & $\theta=30^{\circ}$ & $\theta=45^{\circ}$ & $\theta=60^{\circ}$ \\
\hline \hline$v_{e}$ (STD) & 0 & 0.002 & 0.001 & 0.0015 & 0.0015 \\
\hline$v_{\mu}$ (STD) & 0.0095 & 0.027 & 0.03 & 0.0285 & 0.047 \\
\hline$v_{e}$ (HQ) & 0.2985 & 0.6095 & 0.632 & 0.603 & 0.648 \\
\hline$v_{\mu}$ (HQ) & 0.2945 & 0.6235 & 0.624 & 0.644 & 0.654 \\
\hline
\end{tabular}

Table 1: Results for the average number of electron and muon neutrinos predicted at the sea level, derived using the STD and HQ versions of the CORSIKA Monte Carlo, assuming different values of the zenithal angle.

\section{Conclusions}

In this contribution we present our results for the energy and angular distributions of muons, electron and muon neutrinos. We have modified the CORSIKA Monte Carlo and included the production and propagation of heavy particles in the development of the EAS. Two different models for the heavy quark production have been considered. As a consequence, it was possible to estimate the contribution of prompt leptons, generated by the decay of heavy hadrons. In comparison to the standard CORSIKA predictions, the HQ version implies an enhancement of the lepton fluxes at 

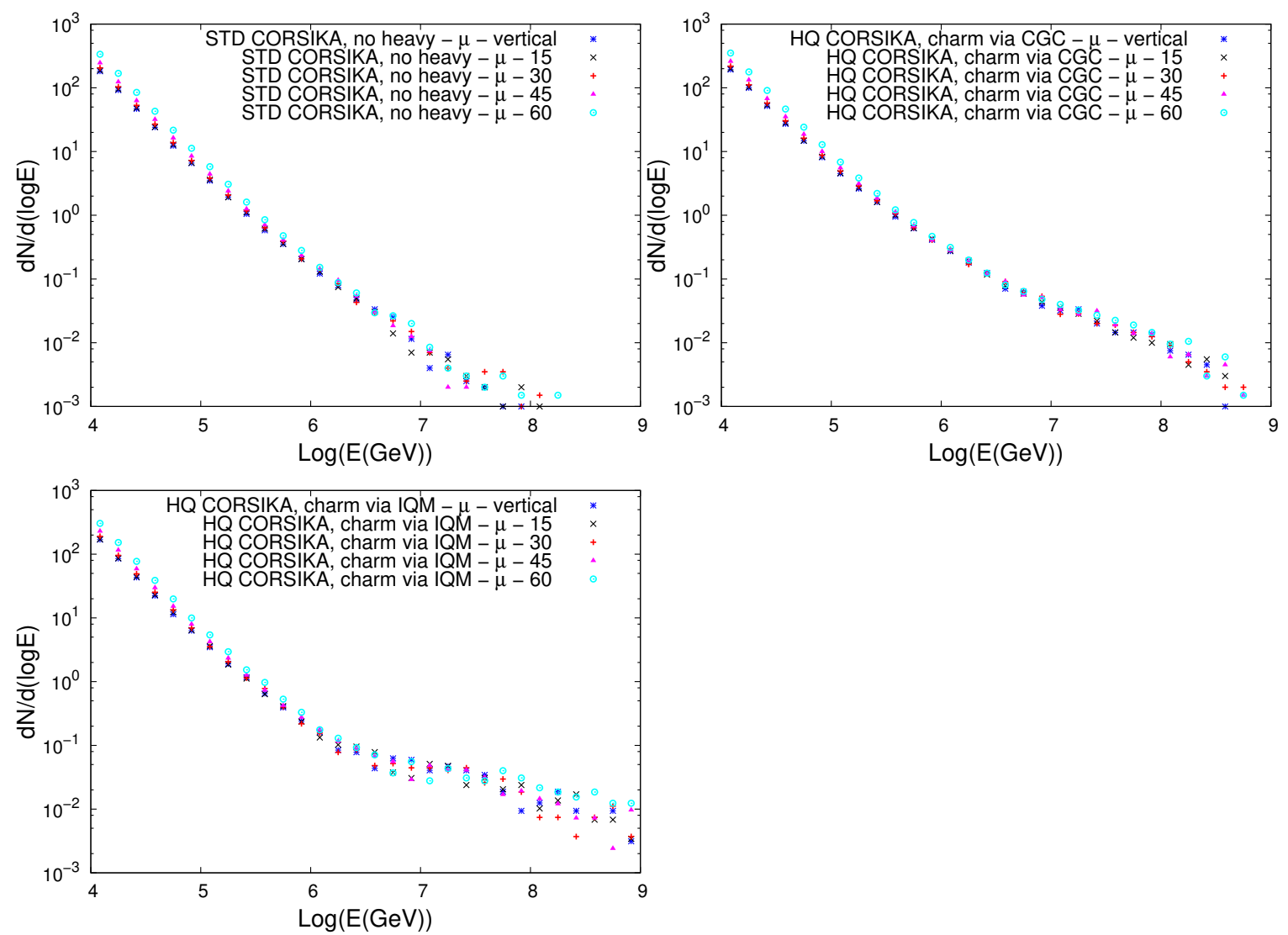

Figure 3: Energy distribution for the prompt $\mu$ flux disregarding the heavy particles in the EASs development (top left panel) and including these particles considering the CGC formalism (top right panel) and Intrinsic Quark Model (lower panel) to describe the heavy quark production. Results for 1000 EASs.

$\mathrm{PeV}$ energies, which is almost independent of the formalism used to describe the heavy quark production. In particular, we predict an excess of the neutrino flux of a factor larger than 15 for very high energies. Moreover, regarding to the angular distribution to the muon and electron neutrinos, our results corroborate that the these neutrinos have the same zenithal angle that the primary particle. A more detailed analysis will be presented in Ref. [15].

\section{Acknowledgments}

We would like to thank Diego Gratieri for the productive discussions. This work was partially financed by the Brazilian funding agencies CNPq, CAPES, FAPERGS and INCT-FNA (process number 464898/2014-5).

\section{References}

[1] V. Agrawal, T. K. Gaisser, P. Lipari and T. Stanev, Phys. Rev. D 53, 1314 (1996)

[2] G. Battistoni, A. Ferrari, P. Lipari, T. Montaruli, P. R. Sala and T. Rancati, Astropart. Phys. 12, 315 (2000) 
[3] C. A. Garcia Canal, J. I. Illana, M. Masip and S. J. Sciutto, Astropart. Phys. 46 (2013) 29

[4] J. I. Illana, P. Lipari, M. Masip and D. Meloni, Astropart. Phys. 34, 663 (2011)

[5] R. Enberg, M. H. Reno and I. Sarcevic, Phys. Rev. D 78, 043005 (2008)

[6] Heck, D., Knapp, J., Capdevielle, J. N., Schatz, G., and Thouw, T., Report FZKA 6019 (1998), Forschungszentrum Karlsruhe, Germany.

[7] A. Bueno and A. Gascon, Comput. Phys. Commun. 185, 638 (2014)

[8] M. A. Muller and V. P. Goncalves, Int. J. Mod. Phys. A 34, no. 12, 1950069 (2019)

[9] M. G. Aartsen et al. [IceCube Collaboration], Science 342, 1242856 (2013).

[10] M. G. Aartsen et al. [IceCube Collaboration], Phys. Rev. Lett. 113, 101101 (2014).

[11] M. G. Aartsen et al. [IceCube Collaboration], Astrophys. J. 833, no. 1, 3 (2016).

[12] V. P. Goncalves and M. V. T. Machado, JHEP 0704, 028 (2007)

[13] F. Carvalho, A. V. Giannini, V. P. Goncalves and F. S. Navarra, Phys. Rev. D 96, no. 9, 094002 (2017)

[14] A. V. Giannini, V. P. Goncalves and F. S. Navarra, Phys. Rev. D 98, no. 1, 014012 (2018)

[15] M. A. Muller and V. P. Gonçalves, Prompt muons and neutrinos in simulations of Extensive Air Showers, in preparation. 